\title{
Proposal for soft-X-ray and XUV lasers in capillary discharges
}

\author{
J. J. Rocca, D. C. Beethe, and M. C. Marconi \\ Department of Electrical Engineering, Colorado State University, Fort Collins, Colorado 80523
}

Received December 29, 1987; accepted April 12, 1988

\begin{abstract}
Capillary plasmas with large length-to-diameter ratios $(1 / d>100)$ are proposed as amplification media for soft- $x$ ray and XUV radiation by direct discharge excitation. The capillary geometry provides a small volume and an adequate resistance for ohmic heating. Heat conduction to the capillary walls provides rapid cooling of the plasma during the decay of the excitation pulse, resulting in a large recombination rate and a population inversion. A timedependent collisional-radiative model of the capillary plasma predicts gains of the order of $5 \mathrm{~cm}^{-1}$ in the $18.2-\mathrm{nm}$ line of C VI.
\end{abstract}

Recently soft-x-ray lasers have been demonstrated by using dense plasmas created by high-energy lasers as the gain medium. ${ }^{1,2}$ In these successful experiments, electron-ion recombination played an important role in achieving the population inversion. ${ }^{1,3}$ Other schemes suitable for short-wavelength amplification have been demonstrated at wavelengths near 100 $\mathrm{nm} .^{4-6}$ All of them use a laser as the pumping source.

In this Letter we discuss the possibility of creating soft-x-ray lasers in recombining capillary plasmas created by a fast discharge. The capillary geometry that we propose allows the creation of plasmas with the characteristics required to explore the amplification of soft $\mathrm{x}$ rays and XUV radiation by using a discharge. The capillary advantages relate to the requirements for the generation of a dense highly ionized plasma as well as its subsequent rapid cooling. Both conditions are necessary to achieve the large recombination rates associated with the gains required in the soft-x-ray and XUV regions of the spectrum, where high-reflectivity resonant cavities are not yet available. In addition, the capillary limits the plasma radius, helping to avoid self-absorption of the lower-level transition.

In this scheme a dense and nearly totally ionized plasma with a large length-to-diameter ratio $(1 / d>$ 100 ) is created from a fast discharge by ionizing material ablated from the capillary walls and from gas that might be introduced into the capillary. Subsequent rapid plasma cooling by heat conduction to the capillary walls, enthalphy loss by particle diffusion, and radiation results in the recombination of highly ionized species into ions of lower charge, creating population inversions.

Several types of discharge have been used in the excitation of relatively low-density plasmas $\left(N_{e}<10^{15}\right.$ $\mathrm{cm}^{-3}$ ) to obtain visible and infrared recombination laser radiation. They include axial and transverse glow discharges, ${ }^{7,8}$ sparks, ${ }^{9}$ arcs, ${ }^{10}$ electron-beam glow discharges, ${ }^{11}$ and flowing negative glows. ${ }^{12}$

To achieve high gain at soft-x-ray and XUV wavelengths by electron-ion recombination requires plasmas that are initially more dense and more highly ionized. The capillary geometry provides a small plasma volume and an adequate resistance for ohmic heating. ${ }^{13}$ The discharge energy required to explore amplification as a result of electron-ion recombination in the $n=3$ to $n=2$ transition of hydrogenic ions with $Z$ up to 6 is relatively modest in a capillary structure. A fast transmission line with an energy of less than $100 \mathrm{~J}$ should be sufficient to create the initial plasma conditions necessary to achieve amplification in the 18.2-nm line of the C VI in a capillary of 20-100 $\mu \mathrm{m}$ in diameter and a few centimeters in length. As a consequence, a compact device could result.

A more significant characteristic of the capillary geometry is the rapid plasma cooling rate achieved by the high heat conduction rate from the plasma volume to the capillary walls. In the majority of the soft-x-ray amplification experiments in laser-generated plasmas, the plasma is created by ablation of the target and cools because of radiative losses ${ }^{1}$ or adiabatic expansion. ${ }^{14,15}$ Ablation targets optimized to achieve cooling by expansion commonly consist of small- (5-7- $\mu \mathrm{m})$ diameter fibers. ${ }^{16}$ In a laser experiment in which a large-diameter fiber was used, heat conduction from the laser-created plasma to the remaining cold core of the 35- $\mu \mathrm{m}$-diameter fiber was observed to be a more important cooling mechanism than expansion or radiation cooling. ${ }^{17}$ A gain of $6 \mathrm{~cm}^{-1}$ was measured in this experiment for the 18.2 -nm transition of C VI. ${ }^{17}$ Conduction cooling is maximized in a capillary structure, since the plasma is closely surrounded by walls that provide a heat sink. In a hot plasma of such a geometry, conduction cooling can be significantly greater than radiation cooling, as shown by the results of calculations done with a time-dependent collisional-radiative plasma mode. Both mechanisms together cause rapid cooling of the plasma during the decay of the discharge pulse.

The model calculates the evolution of the plasma parameters and the time history of the populations of all ionic ground states and relevant excited states. The population densities are calculated from expressions describing the collisional and radiative processes in the plasma and the outflow of charged particles from the plasma volume due to diffusion. A neutral 
atom inflow is also assumed, resulting from ions that recombine at capillary walls. To calculate amplification in the $n=3$ to $n=2$ transition of hydrogenic ions, these ions are modeled with all excited states having principal quantum numbers $n$ up to 12 . The heliumlike ions and the lithiumlike ions are modeled with all states up to $n=6$. Ions with lower charges get ionized shortly after the initiation of the discharge pulse and consequently are modeled with only the ground state. The amount of mass available for ionization is assumed to be constant. A more elaborate calculation would also include mass variation, as material has been ablated from the walls.

The processes considered to transfer populations between those states are electron impact ionization, excitation and deexcitation, three-body and radiative recombination, and radiative decay. Atomic transition probabilities by Wiese et al. ${ }^{18,19}$ and Cohen and McEachran ${ }^{20}$ are used. The collisional rate constants are taken from the literature, ${ }^{21-23}$ and the rates of the reverse processes are calculated by using the principle of detailed balance.

To evaluate the electron temperature evolution we take into account the energy gained by the electron gas owing to ohmic heating, losses due to heat conduction, enthalpy loss due to particle diffusion, and what are usually described as radiation losses. Electron heating due to the reverse collisional processes and to the energy exchange between the electron gas and the ion gas is also considered. The electron and ion transport coefficients are computed according to the expressions derived by Braginskii. ${ }^{24}$ In a stationary plasma the power radiated is equivalent to the power lost by the electron gas owing to collisions. In a transient plasma such as the recombining capillary plasma near the end of the excitation pulse, the power radiated is no longer a good representation of the instantaneous collisional cooling rate of the electron gas. In this case part of the radiated power results from the emission of levels excited by recombination from higher ions that store potential energy from an earlier stage of the discharge. Consequently we make a direct evaluation of the power lost by the electron gas in electron impact ionization, excitation, and bremsstrahlung collisions as well as the heating resulting from the reverse processes instead of by using a radiative power loss coefficient in the electron temperature equation.

While the capillary discharge recombination plasma scheme could be used to amplify several soft- $x$-ray and XUV transitions, we limit our discussion to amplification in the 18.2-nm line of C VI. Figure 1 shows the calculated evolution of the plasma density and the electron and ion temperatures in a carbon capillary plasma $100 \mu \mathrm{m}$ in diameter and $2 \mathrm{~cm}$ in length, assumed to be optically thin to resonant radiation in the radial direction. The discharge power pulse is calculated by a self-consistent solution of the circuit equation with the equations of the parameters that determine the evolution of the plasma resistivity. ${ }^{25}$ The excitation pulse shown in Fig. 1 could be obtained by discharging a $0.15-\mathrm{nF}$ capacitor charged to $700 \mathrm{kV}$. The inductance was assumed to be $0.1 \mu \mathrm{H} .{ }^{26}$ Discharges in polyethelene capillaries $2 \mathrm{~cm}$ in length and diameters as small as $20 \mu$ m have already been demonstrated by using $600-\mathrm{kV}$ excitation. ${ }^{27}$

Figure 1 shows that the electron density reaches a maximum value of $2.4 \times 10^{19} \mathrm{~cm}^{-3}$ in $6.5 \mathrm{nsec}$ after the initiation of the discharge power pulse. The electron temperature reaches a peak value of $720 \mathrm{eV}$ and then decays rapidly because of electron heat conduction and collisional losses. The evolution of the ion temperature follows that of the electron temperature, but with a time lag.

The contribution of the different processes influencing the plasma cooling rate are illustrated in Fig. 2. Electron heat conduction to the walls of the capillary is the dominant plasma-cooling mechanism. During the first part of the current pulse, the power lost by the electron gas owing to ionizing and exciting collisions is of the same order as the conduction losses, while heating due to electron-ion recombination is negligible. As the current pulse decreases, the self-generated

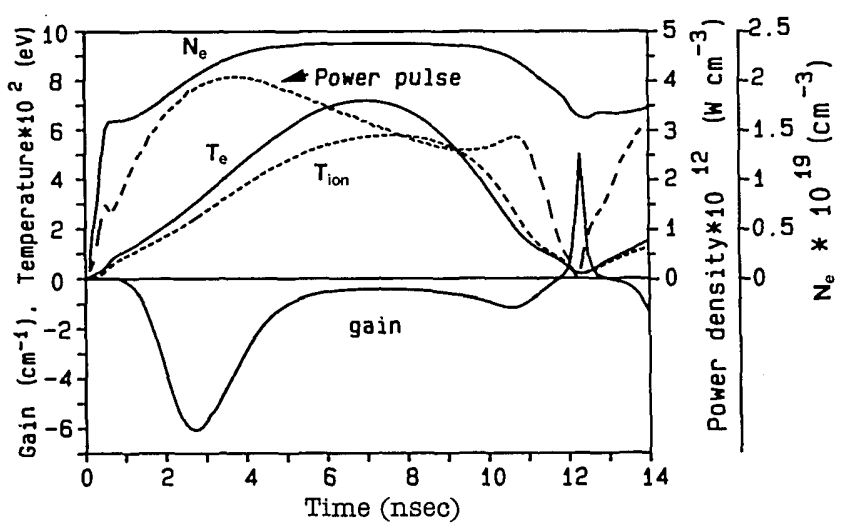

Fig. 1. Temporal evolution of the plasma density $\left(N_{e}\right)$ and electron $\left(T_{e}\right)$ and ion $\left(T_{\text {ion }}\right)$ temperatures in a carbon capillary discharge $100 \mu \mathrm{m}$ in diameter and $2 \mathrm{~cm}$ in length excited by the power pulse shown. The plasma is assumed to be optically thin in the radial direction. The number of carbon atoms assumed to be available for ionization is $4 \times 10^{18} \mathrm{~cm}^{-3}$. The predicted gain in the C VI 18.2-nm transition is also shown.

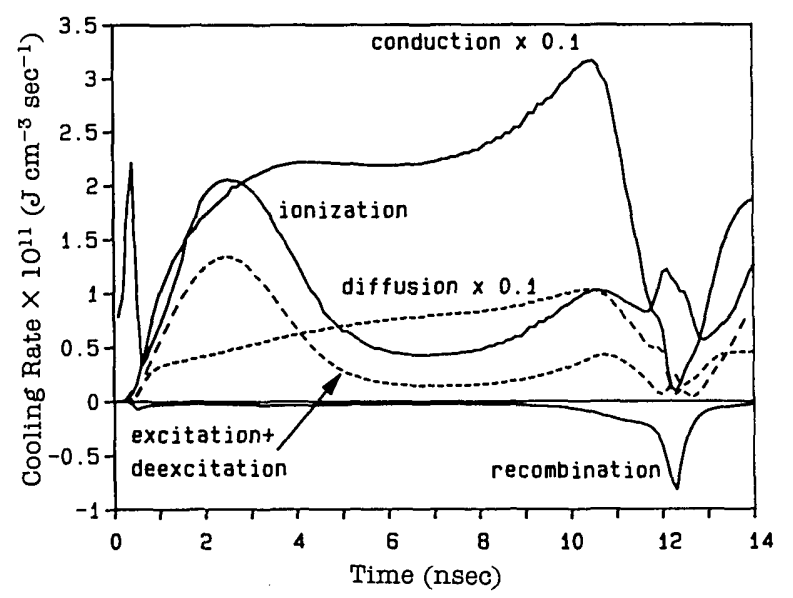

Fig. 2. Evolution of the physical processes that govern the plasma cooling. Negative values represent heating of the electron gas. 


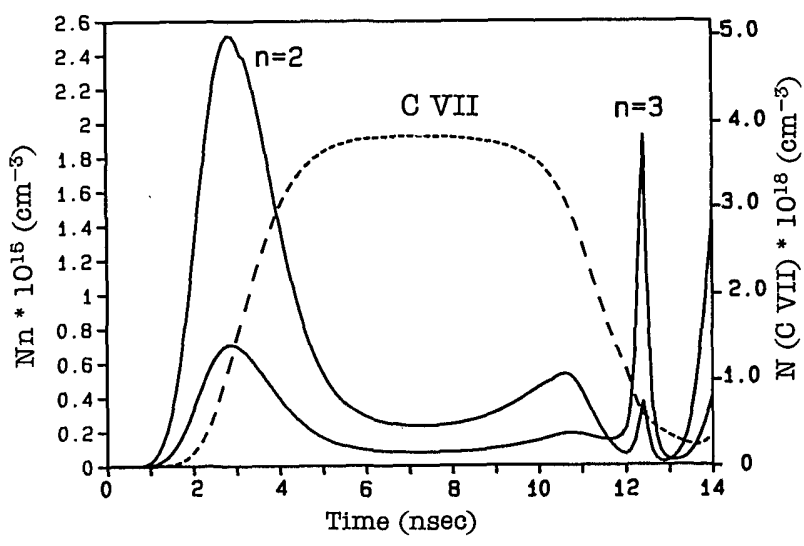

Fig. 3. Temporal evolution of the concentration of the bare nucleus (C VII) and the populations of the $n=2$ and $n=3$ levels of $C$ VI for a $100-\mu \mathrm{m}$-diameter carbon capillary plasma excited under the conditions described in Fig. 1. The corresponding gain coefficient for the C VI 18.2-nm line is the one shown in Fig. 1.

magnetic field also drops and thus the electron conduction losses increase to become significantly larger than other heat-loss mechanisms.

Figure 2 shows that as the plasma temperature decreases, recombination heating increases. The maximum population inversion between levels $n=3$ and $n=2$, illustrated in Fig. 3 , occurs when the electron temperature reaches $17.8 \mathrm{eV}$. At this point the gain in the $18.2-\mathrm{nm} \mathrm{C}$ VI line is $5 \mathrm{~cm}^{-1}$, assuming Doppler broadening, and corresponds to an exponential gain of $2.2 \times 10^{4}$. Subsequently the rise of the discharge current during the second half-cycle causes the termination of the amplification as the plasma heats up again. The calculations show that suppression of the current ringing would result in gains greater than 10 $\mathrm{cm}^{-1}$.

In summary, capillary plasmas are shown to be adequate media for the amplification of soft- $x$-ray radiation by direct discharge excitation. The capillary geometry provides rapid cooling of the plasma by electron heat conduction to the walls. Large recombination rates result in inversions of the order of magnitude required to obtain laser radiation in soft-x-ray and XUV spectral regions.

We want to acknowledge David Kahaner of the Center for Applied Mathematics, National Bureau of Standards, for his assistance in the solution of the system of nonlinear differential equations. We also want to acknowledge the generous cooperation of David Palermo, MSO laboratory manager at HewlettPackard, Loveland, Colorado, for providing the computational resources for the development of this model. This research was supported by National Science Foundation Grant no. ECS-8606226 and a National Science Foundation Presidential Young Investigator Award (to J. J. Rocca). M. C. Marconi was supported by a fellowship from the Universidad Nacional de
Buenos Aires and the Consejo Nacional de Investigaciones Cientificas y Tecnicas de la Republica Argentina.

\section{References}

1. S. Suckewer, C. H. Skinner, H. Milchberg, and D. Vorhees, Phys. Rev. Lett. 55, 1973 (1985).

2. D. L. Matthews, P. L. Hagelstein, M. D. Rosen, M. J. Eckart, N. M. Ceglio, A. V. Hazi, H. Medecki, B. J. MacGowan, J. E. Trebes, B. L. Whitten, E. M. Campbell, C. W. Hatcher, A. M. Hawryluk, R. L. Kauffman, L. D. Pleasance, G. Rambach, J. H. Scofield, G. Stone, and T. A. Weaver, Phys. Rev. Lett. 54, 110 (1985).

3. J. P. Apruzese, J. Davis, M. Blaha, P. C. Kepple, and V. L. Jacobs, Phys. Rev. Lett. 55, 1877 (1985).

4. K. Boyer, H. Egger, T. S. Luk, H. Pummer, and C. K. Rhodes, J. Opt. Soc. Am. B 1, 3 (1984).

5. H. C. Kapteyn, R. W. Lee, and R. W. Falcone, Phy. Rev. Lett. 57, 2939 (1986).

6. M. H. Sher, J. J. Macklin, J. F. Young, and S. E. Harris, Opt. Lett. 12, 891 (1987).

7. V. Zhukov, E. Latush, V. Mikhalewskii, and S. Sem, Sov. J. Quantum Electron. 7, 407 (1977).

8. M. S. Butler and J. A. Piper, Appl. Phys. Lett. 42, 1008 (1983).

9. S. Silfvast, L. Szeto, and O. Wood II, Appl. Phys. Lett. 36, 615 (1980).

10. S. Silfvast, O. Wood II, and J. Macklin, Appl. Phys. Lett. 42, 347 (1983).

11. J. J. Rocca, H. Mancini, and B. Wernsman, IEEE J. Quantum Electron. QE-22, 509 (1986).

12. J. J. Rocca, Appl. Phys. Lett. 47, 1145 (1985).

13. R. A. McCorkle, Ann. N.Y. Acad. Sci. 342, 53 (1980); P. Bogen, H. Conrads, G. Gatti, and W. Kohlhaas, J. Opt. Soc. Am. 58, 203 (1968).

14. D. Jacoby, G. Pert, S. A. Ramsden, L. D. Shorrock, and G. J. Tallents, Opt. Commun. 38, 193 (1981).

15. D. Jacoby, G. J. Pert, L. D. Shorrock, and G. J. Tallents, J. Phys. B 15, 3557 (1982).

16. G. J. Pert, J. Opt. Soc. Am. B 4, 602 (1987).

17. H. Milchberg, C. H. Skinner, S. Suckewer, and D. Voorhees, Appl. Phys. Lett. 47, 1151 (1985).

18. W. L. Wiese, M. W. Smith, and B. M. Glennon, Atomic Transitions Probabilities (U.S. Government Printing Office, Washington, D.C., 1966), Vol. 1.

19. G. J. Martin and W. L. Wiese, J. Phys. Chem. Ref. Data 5, 537 (1976).

20. M. Cohen and R. P. McEachran, Can. J. Phys. 50, 1363 (1972).

21. M. Seaton, in Atomic and Molecular Processes, D. R. Bates, ed. (Academic, New York, 1962), p. 374.

22. H. Kunze, Phys. Rev. A 3, 937 (1971).

23. M. J. Seaton, Mon. Not. R. Astron. Soc. 119, 81 (1959).

24. S. I. Braginskii, in Reviews of Modern Physics (Consultants Bureau, New York, 1965), Vol. 1.

25. L. Spitzer Jr., Physics of Fully Ionized Gases (Wiley, New York, 1962).

26. W. C. Nunnally, L. A. Jones, and S. Singer, in Digest of Technical Papers of the IEEE International Pulsed Power Conference (Institute of Electrical and Electronics Engineers, New York, 1979), p. 142.

27. D. R. Kania and L. A. Jones, in Digest of the IEEE International Conference on Plasma Science (Institute of Electrical and Electronics Engineers, New York, 1984). 